\title{
EXPERIMENTAL STUDY OF STRUCTURAL ZONE MODEL FOR COMPOSITE THIN FILMS IN MAGNETIC RECORDING MEDIA APPLICATION
}

\author{
Hua Yuan and David E. Laughlin
}

Department of Materials Science and Engineering, Carnegie Mellon University, Pittsburgh, PA 15213, USA and the Data Storage Systems Center, Carnegie Mellon University, Pittsburgh, PA 15213, USA

\begin{abstract}
Composite thin films are a new area of study with many applications, e.g. metal + oxide thin films in high density magnetic recording media. Engineering the processing, microstructure and properties of these thin films is of great importance. It has been found that microstructures of the thin films depend strongly on the oxide volume fraction and pressure during sputtering. Surface diffusion and self-shadowing effects are found to play important roles in determining various thin film microstructures under different processing conditions. Four characteristic microstructural zones could be generally distinguished: "percolated type"(A), "maze-like type"(T), "granular columnar type”(B) and “embedded type”- a microstructure with metal nanocrystals embedded in the amorphous matrix (C). This modified structural zone model of composite thin films has been proposed as a supplement to Thornton's model for sputtered thin film system.
\end{abstract}

This research has been sponsored by Seagate Technology and the DSSC of CMU.

\section{INTRODUCTION}

Composite thin films e.g. metal + oxide thin films are widely used in high density magnetic recording media. ${ }^{1-4}$ These films are composed of the crystalline metallic phase and a secondary amorphous phase. Engineering the processing, microstructure and properties of these thin films is of great importance. The studies about composite thin films are limited and nonsystematic in literature. In addition, the real microstructures of composite thin films can be very complicated by varying various factors. Even a slight change of microstructure could cause a significant degradation of film properties. As a result, it is important to have a comprehensive understanding of how the composite thin films grow and how their microstructures evolve during sputter deposition, especially for composite thin films fabricated at room temperature for current perpendicular magnetic recording media. In order to predict the microstructures of composite thin films, it will be informative to construct a phenomenological structural zone model which includes the relative volume fractions of the two phases present. This supplements Thornton's structural zone models for the sputter deposited thin films. In this paper, the major results based on the composite $\mathrm{Ru}+$ oxide thin films for magnetic recording media application will be discussed in details.

It has been found that microstructures of the thin films depend strongly on the oxide volume fraction and pressure during sputtering. Surface diffusion and self-shadowing effects are found to play an important role in determining various thin film microstructures under different processing conditions. Four characteristic microstructural zones could be generally distinguished: "percolated type”(A), “maze-like type”(T), "granular columnar type”(B) and 
"embedded type"- a microstructure with metal nanocrystals embedded in the amorphous matrix (C). This modified structural zone model of composite thin films has been proposed as a supplement to Thornton's model for sputtered thin film system.

\section{EXPERIMENT}

Thin films: glass substrate $\backslash \mathrm{Ta}(3 \mathrm{~nm}) \backslash \mathrm{Ru}$ (low pressure, $15 \mathrm{~nm}) \backslash \mathrm{Ru}+\mathrm{TiO}_{2}(10 \mathrm{~nm}) \backslash$ CoPt + oxide $(11 \mathrm{~nm}) \backslash$ carbon overcoat was sputter deposited at room temperate. Ta \Ru seedlayers are utilized to provide good adhesion and (00.2) crystallographic texture for the composite $\mathrm{Ru}+\mathrm{TiO}_{2}$ layer. $\mathrm{Ru}+\mathrm{TiO}_{2}$ interlayer was studied at three pressures: $3 \mathrm{mT}, 30 \mathrm{mT}$ and $60 \mathrm{mT}$. The volume fraction of $\mathrm{TiO}_{2}$ varied from 0 vol \% to $50 \mathrm{vol} \%$ in the $\mathrm{Ru}+$ oxide interlayer. The magnetic layer in each sample was fixed with the same composition and thickness. Hysteresis loops were measured by the magneto-optic Kerr effect magnetometer (MOKE). The crystalline orientation was evaluated from X-ray rocking curve scans of Ru (00.2) peaks taken on a Philips X'pert diffractometer. Microstructures and surface morphology of samples deposited up to composite $\mathrm{Ru}+\mathrm{TiO}_{2}$ layer and samples up to composite CoPt + oxide magnetic layer were analyzed on the JOEL 2000 transmission electron microscope (TEM).

\section{RESULTS AND DISCUSSION}

By means of varying the oxide volume fraction and pressure during sputtering, the microstructure of composite $\mathrm{Ru}+\mathrm{TiO}_{2}$ thin films underwent a significant change. TEM bright field imaging technique has been used to observe the microstructure features of the thin films in the study. The morphologies of such thin films are schematically drawn in Fig. 1, where the dark area represents for the crystalline metal phase and white area represents for the amorphous secondary phase region (e.g. oxide). Four characteristic microstructural zones could be generally distinguished: "percolated"(A), "maze-type”(T), "granular”(B) and "embedded". This is also listed in Table I. Zone A represents the low pressure, low volume fraction (VF) region of the plot, where only the surface diffusion effect is crucial. A continuous metallic film with oxide phase of small feature sizes is commonly observed. The thin films exhibit good crystallinity. The qualitative texture evolution of the composite thin films as a function of oxide volume fraction and pressure is mapped in Fig. 2.

Zone B is for high pressure, low oxide volume fraction region, where the self-shadowing effect overwhelms the surface diffusion effect. A columnar granular microstructure is the typical type of morphology in this region. Within the medium pressure and low oxide volume fraction region, denoted as transition zone $\mathrm{T}$, the thin film microstructure shows networking or maze-like characteristics. Both the surface diffusion and the self-shadowing effects play equivalent roles in determining the final thin film morphology. With an increase in the amorphous phase fraction, the feature size decreases continuously.

Zone $\mathrm{C}$ covers rest of the plot, which is within high oxide volume fraction region and under all pressure range. Here, the metal nanocrystals are dispersed and embedded within the amorphous oxide matrix.

Fig. 3 shows the plan-view TEM bright field micrographs of composite $\mathrm{Ru}+$ oxide thin films with 4 different microstructures. They are located at both low and high oxide volume fraction ends of zone A (percolated) and zone B (granular). It is obvious that the structure zone plot describes well the microstructures of the composite thin films $\left(\mathrm{Ru}+\mathrm{TiO}_{2}\right)$ sputter deposited at different pressure and oxide volume fraction values. Region 1 shows a columnar granular microstructure of Ru grains with voids and oxide rich grain boundaries under high pressure and low oxide volume fraction condition. This is dominated by the enhanced self-shadowing effect. 
Region 2 shows a dense Ru grain structure with low defects density, low voids density and smooth surface. This is mainly determined by the high surface mobility induced by the low pressure and low oxide volume fraction condition. As for region 3, under low pressure and high oxide volume fraction condition, the oxide phase forms in the metal thin film and it shows a percolated microstructure. Fine Ru and oxide grains as small as $2 \sim 3 \mathrm{~nm}$ coexist with up to 40 vol \% oxide addition. The density of the oxide phase increases with increasing oxide amount. Region 4 displays a microstructure of many smaller columnar Ru grains with oxide segregated within the grain boundaries, compared to the microstructure of region 1 . While the grain size becomes smaller, the grain uniformity degrades with increasing oxide amount.

Fig. 4 shows the plan-view TEM bright field micrographs of Co-alloy + oxide thin films that have been deposited on top of composite $\mathrm{Ru}+\mathrm{TiO}_{2}$ thin films with the 4 different microstructures shown in Fig. 3. With increasing oxide volume fraction in the composite $\mathrm{Ru}+$ $\mathrm{TiO}_{2}$ thin films, the CoPt grain size decreases. This demonstrates that the microstructure evolution in the composite underlayer affects the microstructure of the magnetic layer. As for region 1 , the $\mathrm{Ru}$ grain size and $\mathrm{CoPt}$ matches well, implying a one-to-one epitaxial growth of the grains in these two adjacent layers. It is not surprising that the magnetic grain size does not change much on top of region 1 and region 2 microstructures, as not much oxide was added in the $\mathrm{Ru}$ layer. However, the $\mathrm{CoPt}$ grain size on top of $\mathrm{Ru}+\mathrm{TiO}_{2}$ thin film (region 3) of percolated microstructure has been reduced to $5.8 \mathrm{~nm}$. The CoPt grain size on top of $\mathrm{Ru}+\mathrm{TiO}_{2}$ thin film (region 4) of smaller granular non-uniform microstructure has been also reduced to 6.6 nm.

In addition to the effect of the microstructure of the composite $\mathrm{Ru}+\mathrm{TiO}_{2}$ thin films on the microstructure of the magnetic layer, there is also a great effect on the resulting properties of the magnetic layer. Fig. 5 shows the perpendicular $\mathrm{MH}$ loops of Co-alloy + oxide thin films on top of composite $\mathrm{Ru}+$ oxide thin films with 4 different microstructures. Due to the perfect oneto-one epitaxial growth for magnetic film on top of region 1 underlayer, the films show the highest coercivity $\left(\mathrm{H}_{\mathrm{c}}\right)$, the most negative nucleation field $\left(\mathrm{H}_{\mathrm{n}}\right)$, a low $\left(\mathrm{H}_{\mathrm{c}}-\mathrm{H}_{\mathrm{n}}\right)$ value and a high squareness value. This indicates a good inter-granular exchange-decoupling, crystallographic orientation and narrow c-axis distribution in the magnetic layer.

As for the magnetic film on top of the region 2 underlayer, although the microstructure of the magnetic layer is similar to the former case, the grains are highly exchange coupled due to the dense Ru grain structure. This decreases $\mathrm{H}_{\mathrm{c}}$ and $\left(\mathrm{H}_{\mathrm{c}}-\mathrm{H}_{n}\right)$ values. However, squareness of the magnetic film is improved greatly due to the effect of very good crystallographic texture of the composite Ru underlayer, which was deposited under very low pressure and with very low oxide addition.

As the magnetic film is on top of region 3 underlayer, the very small magnetic grain size has decreased the $\mathrm{H}_{\mathrm{c}}$ value. And the degraded texture of the magnetic layer resulting from the percolated underlayer microstructure reduces the squareness.

The magnetic film which grew on top of the region 4 underlayer, the magnetic grain size slightly decreases to $6.6 \mathrm{~nm}$. It is likely that the granular microstructure in the $\mathrm{Ru}+\mathrm{TiO}_{2}$ underlayer is too non-uniform to form a one-to-one epitaxial growth. Therefore, both the smaller magnetic grain size and non-ideal growth decreases the $\mathrm{H}_{\mathrm{c}}$. Nevertheless, due to the segregation of high percentage of oxides in the grain boundaries, the magnetic grains are better exchange decoupled, judging from the hysteresis loops.

\section{CONCLUSION}


Metal + oxide (e.g. $\mathrm{Ru}+\mathrm{TiO}_{2}$ ) composite thin films were chosen as underlayer materials for magnetic recording media due to their unique segregated microstructure. The metal and oxide phases in the composite thin films are immiscible. The final microstructure of the interlayer depends on factors, such as, sputtering pressure, oxide species, oxide volume fraction, thickness, alloy composition, temperature etc. Moreover, it has been found that the microstructure of the composite thin films is affected mostly by two important factors - oxide volume fraction and sputtering pressure. The latter affects grain size and grain segregation through surface-diffusion modification and the self-shadowing effect. The composite $\mathrm{Ru}+$ oxide interlayers were found to have various microstructures under various sputtering conditions. Four characteristic microstructure zones can be identified as a function of oxide volume fraction and sputtering pressure - "percolated”(A), "maze”(T), "granular”(B) and "embedded” (C), based on which, a new structural zone model (SZM) is established for composite thin films. The evolution of microstructures determines the properties of the thin films significantly.

\section{ACKNOWLEDGEMENTS}

The authors would like to thank the financial support by Seagate Technology and Data Storage Systems Center (DSSC) at Carnegie Mellon University. We are grateful for discussions with Dr. Bin Lu, Dr. Yingguo Peng and Prof. Jimmy Zhu.

\section{REFERENCES}

${ }^{1}$ T. Oikawa, M. Nakamura, H. Uwazumi, T. Shimatsu, H. Muraoka, and Y. Nakamura, IEEE Tran. Magn., 38, 1976 (2002).

${ }^{2}$ H. Uwazumi, K. Enomoto, Y. Sakai, S. Takenoiri, T. Oikawa, and S. Watanabe, IEEE Trans. Magn., 39, 1914 (2003).

${ }^{3}$ Y. Inaba, T. Shimatsu, T. Oikawa, H. Sato, H. Aoi, H. Muraoka, and Y. Nakamura, IEEE Trans. Magn., 40, 2486 (2004).

${ }^{4}$ J. Ariake, T. Chiba, and N. Honda, IEEE Trans. Magn., 41, 3142 (2005).

Table I: List of four characteristics structural zones of sputtered composite metal + oxide thin films.

\begin{tabular}{cccc}
\hline Zone & $\begin{array}{c}\text { Pressure } \\
(\mathrm{mT})\end{array}$ & $\begin{array}{c}\text { Oxide volume fraction } \\
(0-100 \%)\end{array}$ & $\begin{array}{c}\text { Structural } \\
\text { characteristics }\end{array}$ \\
\hline $\mathrm{A}$ & low & low - medium & "percolated" \\
$\mathrm{T}$ & medium & low - medium & "maze" \\
$\mathrm{B}$ & high & low - medium & "granular" \\
$\mathrm{C}$ & all & high & "embedded" \\
\hline
\end{tabular}




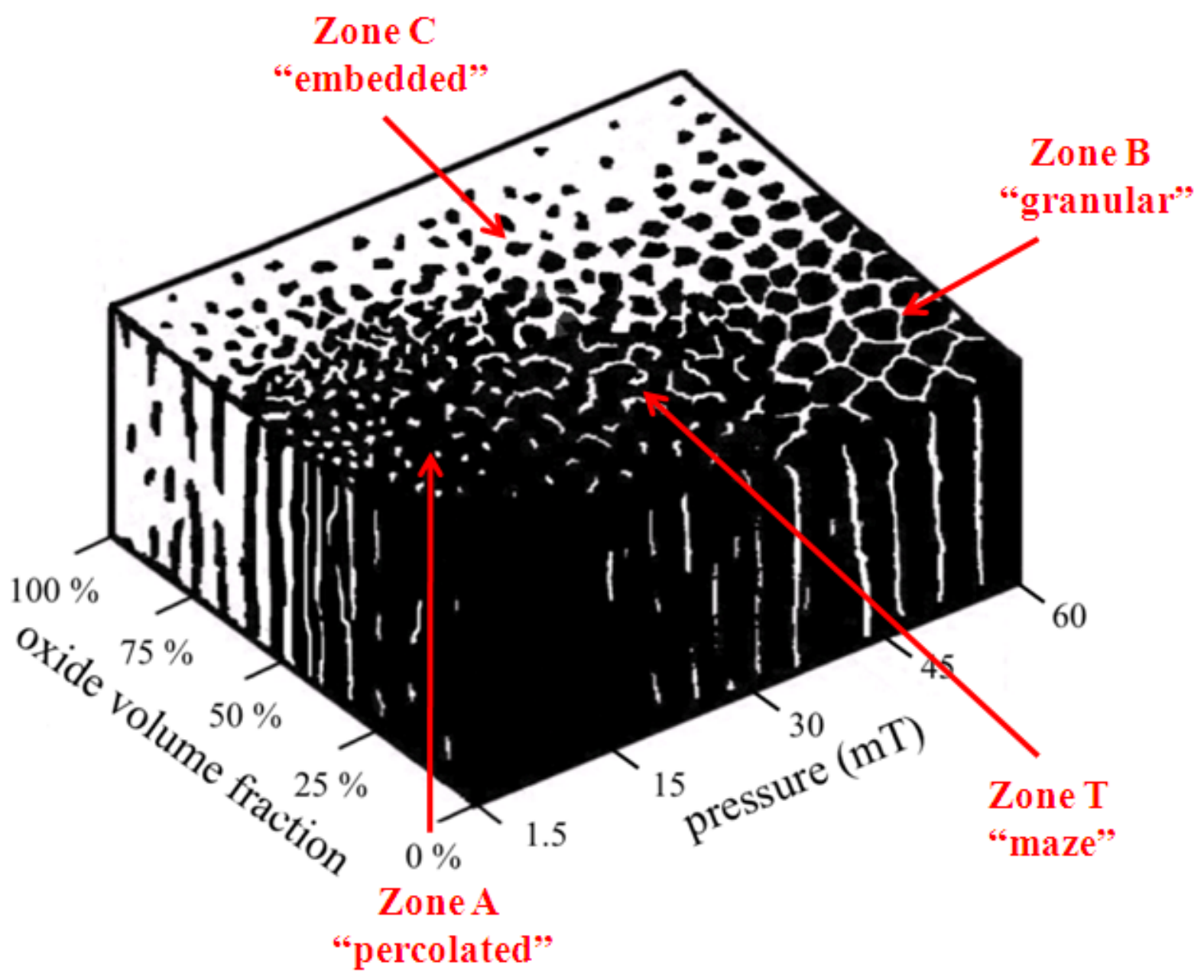

Figure 1 Schematic illustrations of the structural zones of composite metal + oxide thin films as a function of inert Ar gas pressure and secondary phase volume fraction (e.g. oxide volume fraction). Four characteristic zones - "percolated”, "granular”, “ maze” and "embedded” are distinguished. 


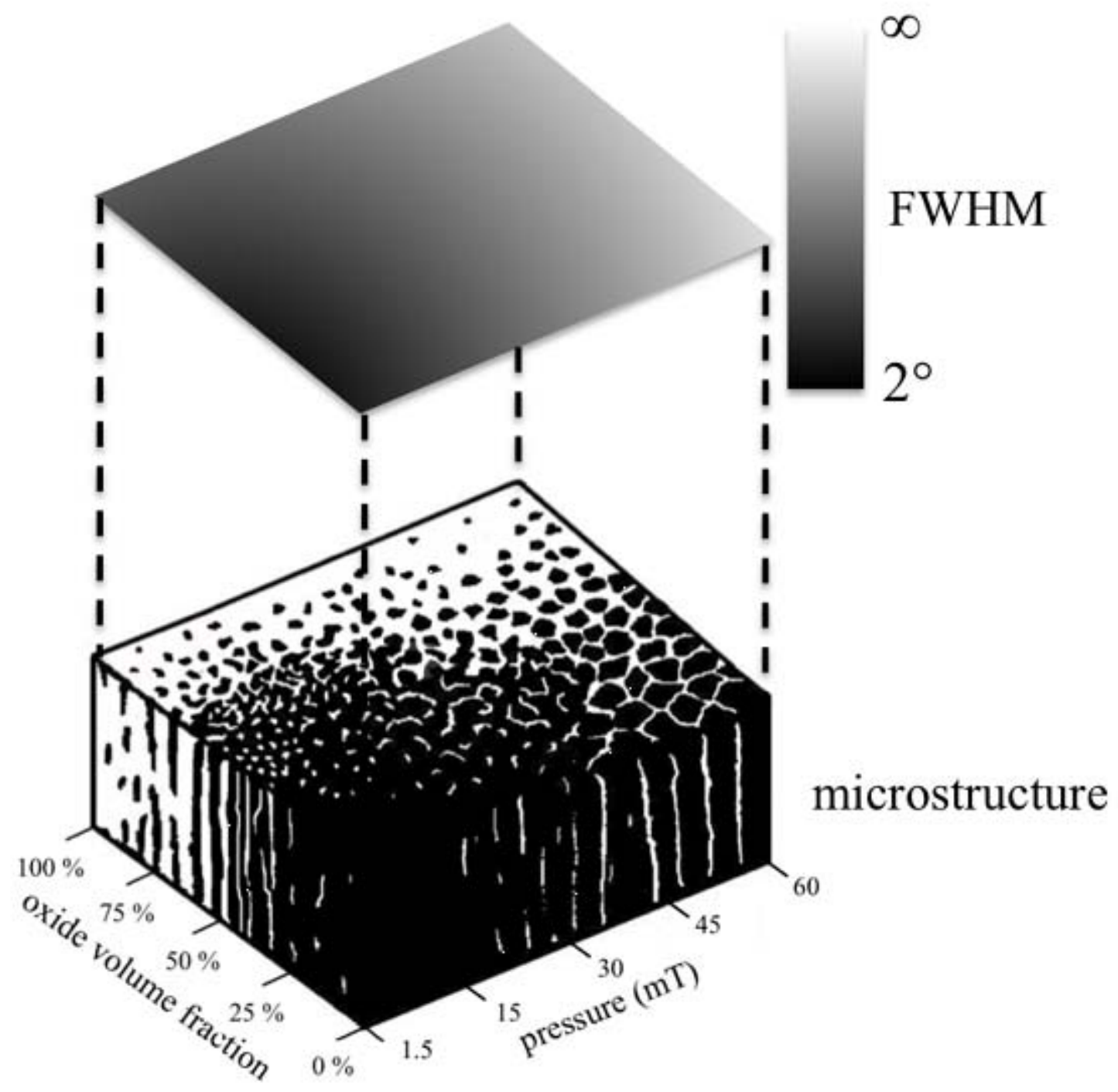

Figure 2 Schematic illustrations of the structural zones and the crystallographic texture of composite metal + oxide thin films as a function of inert Ar gas pressure and secondary phase volume fraction (e.g. oxide volume fraction). 

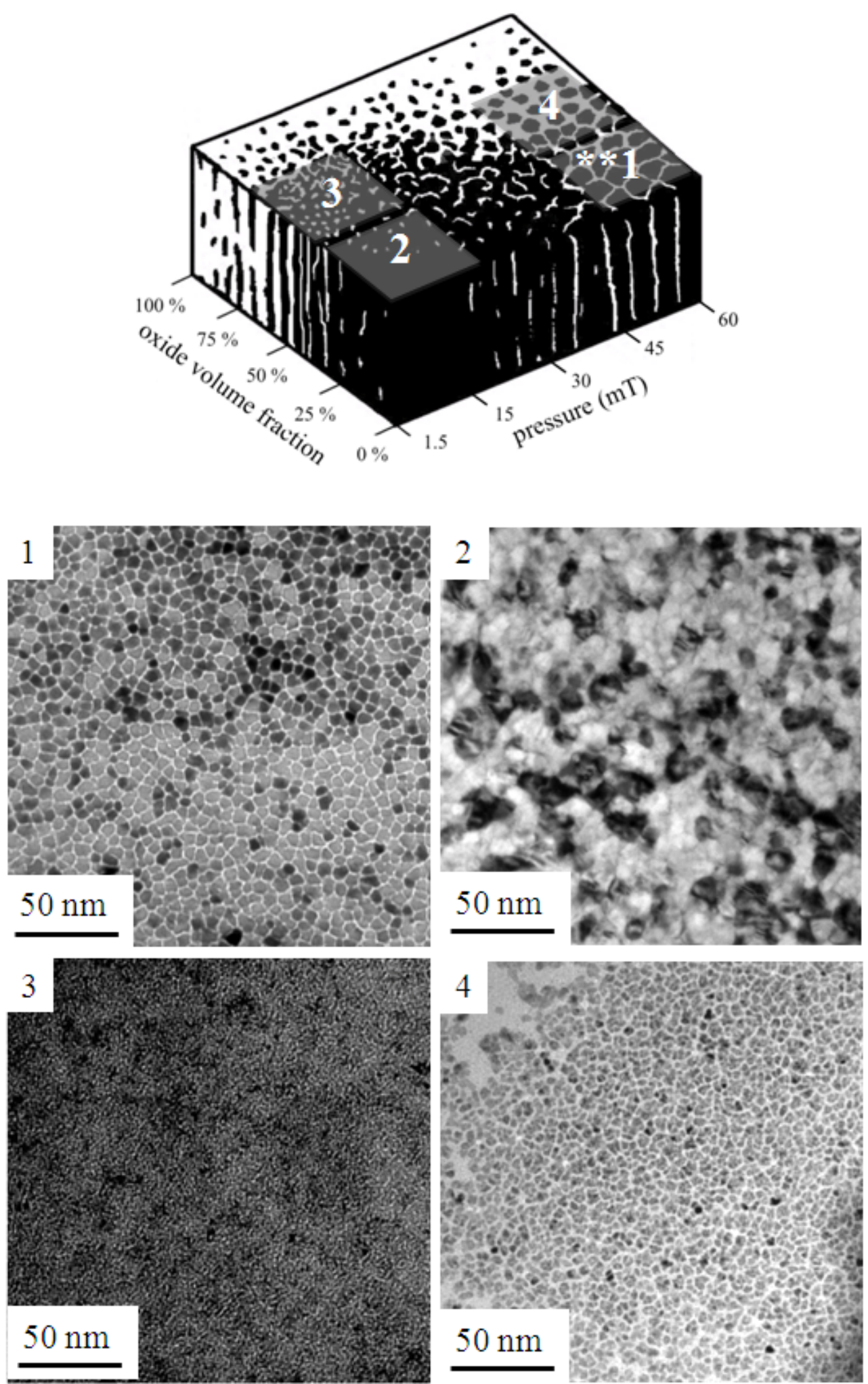

Figure 3 Plan-view TEM bright field micrographs of composite Ru + oxide thin films with 4 different microstructures. 

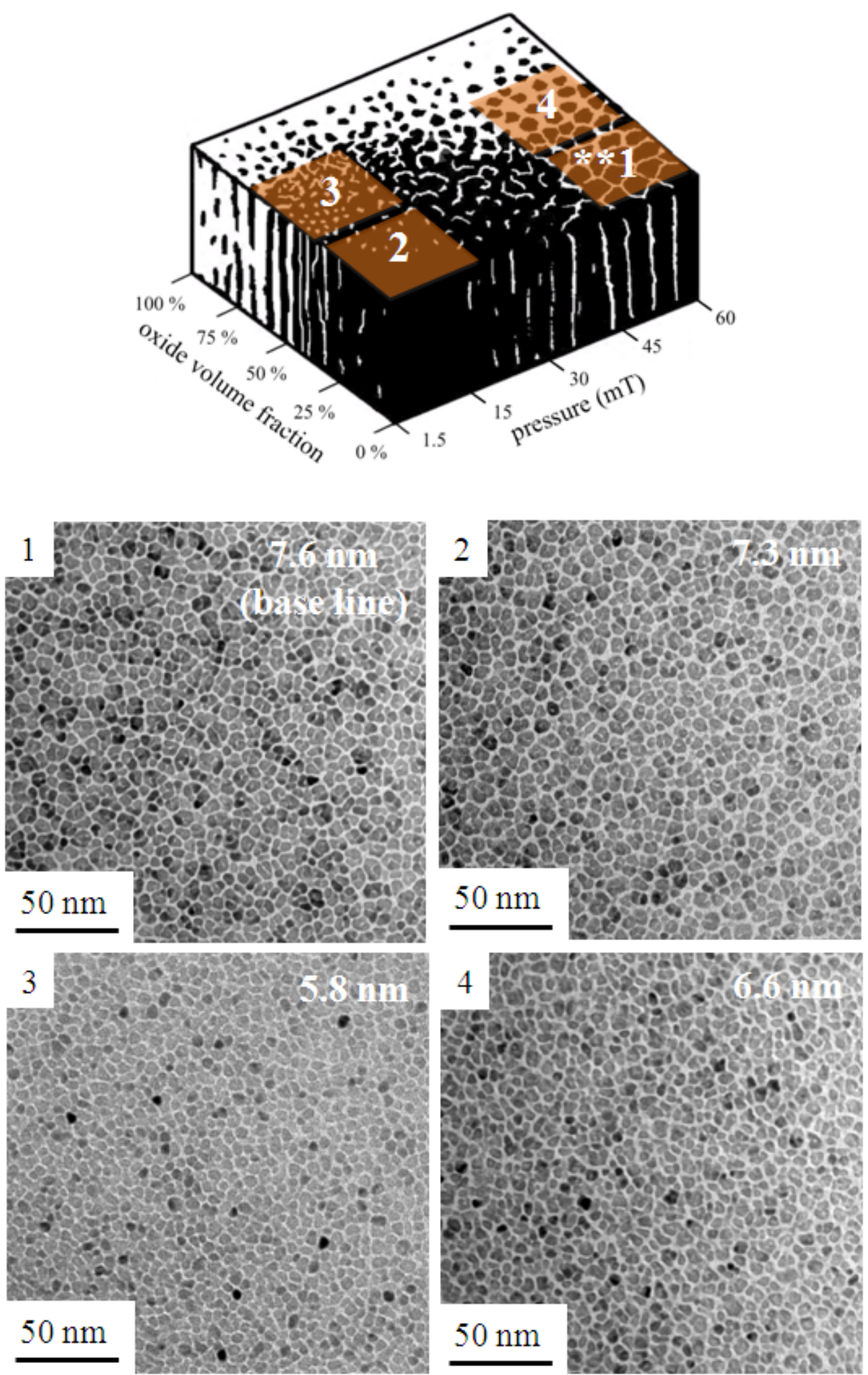

Figure 4 Plan-view TEM bright field micrographs of Co-alloy + oxide thin films on top of composite $\mathrm{Ru}+$ oxide thin films with 4 different microstructures. 

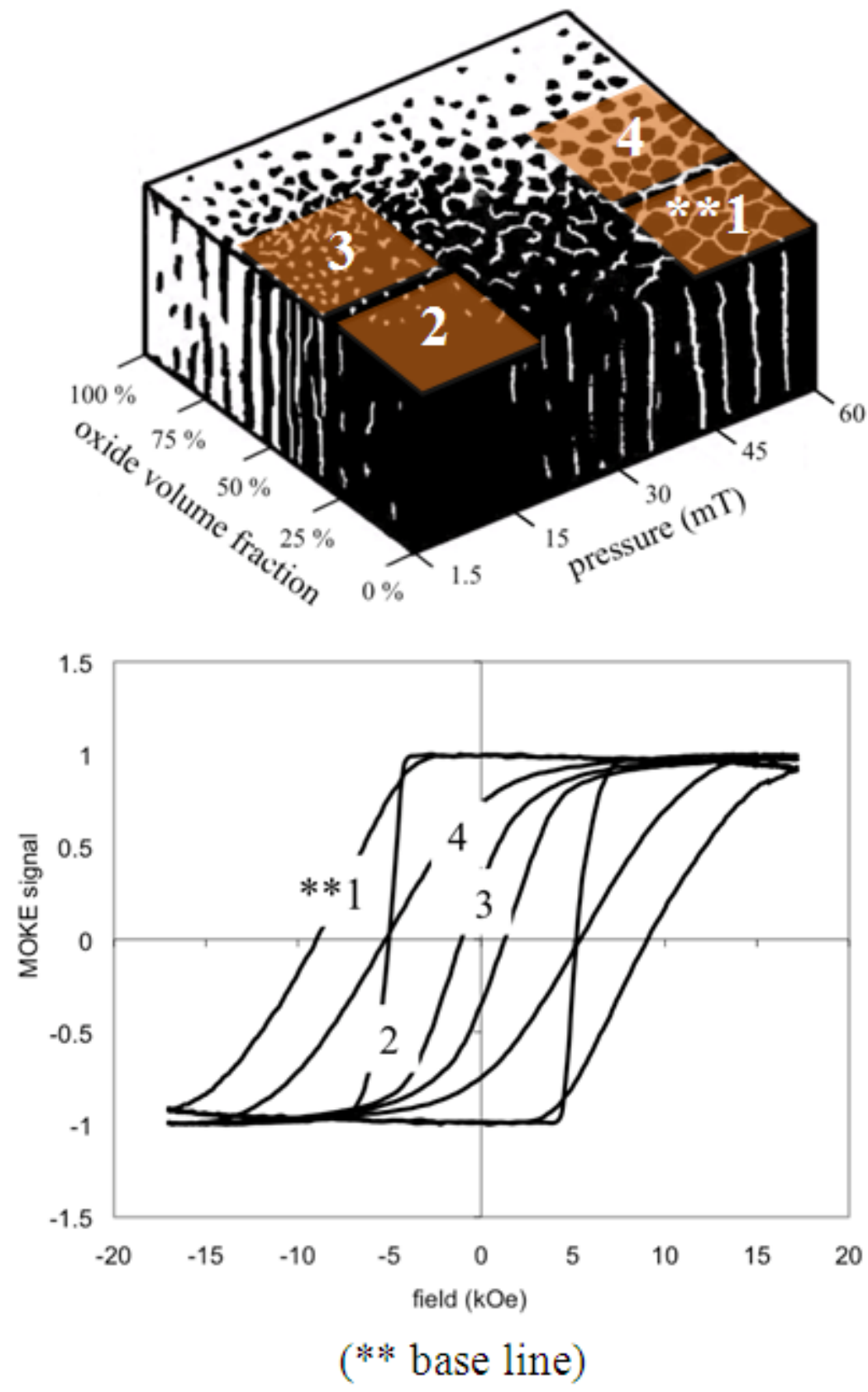

Figure 5 Perpendicular MH loops of Co-alloy + oxide thin films on top of composite $\mathrm{Ru}+$ oxide thin films with 4 different microstructures. 